\title{
Modelling an Intelligent Interaction System for Increasing the Level of Attention
}

\author{
Dalila Durães ${ }^{1,3}$, David Castro ${ }^{2,}$ Javier Bajo $^{1}$, Paulo Novais ${ }^{2}$ \\ ${ }^{1}$ Department of Artificial Intelligence, Technical University of Madrid, Madrid, Spain \\ d.alves@alumnos.upm.es, jbajo@fi.upm.es \\ ${ }^{2}$ Algoritmi Center, Minho University, Braga, Portugal \\ pg30465ealunos.uminho.pt, pjon@di.uminho.pt \\ ${ }^{3}$ CIICESI, ESTGF, Polytechnic Institute of Porto, Felgueiras, Portugal
}

\begin{abstract}
Learning activities using technologies is one of the common education methods. Its advantages allow that students can learn with concepts more practical's. However in this environment not all the students can be attentive. In this research an Ambient Intelligent System has been designed using biometrics behaviors for detecting learner inattentiveness. The learning attentiveness of a student can be determined precisely and the teacher has access to these results and might improve news strategies.
\end{abstract}

Keywords: Level of Attention, Technology in Learning, and Ambient Intelligent Systems.

\section{Introduction}

In a traditional learning environment student and teacher are the key elements in the classroom. In environments in which technologies exist, the teacher plays an essential role in providing an engaging learning and teaching environment. Together, they take a set of physical, social, emotional, mental characteristics, and needs to the classroom. These influence the way one relates with the other and consequently affect the way the learning process will progress. Teacher is able to learn about the necessities and the educational philosophy as well the nature of his/her students; this will improve teacher's position in order to facilitate student's learning.

For these reasons, education, training, skills development, and learning are processes that are continuously performed since we are born. These characteristics and the ability to learn and teach, allow us to grow as a person. The learning concept is described as the act of acquiring knowledge, behaviors, abilities, standards or preferences and the study of learning has been closely linked to the development of psychology as a science [1].

The school must create systems that are capable of involving students and capture their attention. Advances in computers and wireless technologies have also had an impact on the educational setting, thus generating a new approach for Ambient Intelligent (AmI) systems. The rapid development of these technologies combined with 
access to content in a wide variety of settings, allows learners to experience new learning situations beyond the school's wall.

In the last years, school has introduced news technology with a set of computers, networks and access to the Internet in order to improve learning. These systems have implemented two important features: context awareness and adaptively. Context awareness denotes that the pedagogical progress and the context that are provided to the learning environment should be aware of the situations in which the learner actually is. Context of adaptively refers to the idea that different learning context should be adaptable to the particular setting in which the learner is situated [2].

Being a cognitive process, attention is strongly connected with learning [3]. When it comes to acquiring new knowledge, attention can be considered one of the most important mechanisms [4], where the level of the learner's attention affects learning results, and the lack of attention can define the success of a student.

Teaching should be solidly grounded to the absolute understanding of how the process of learning occurs, so that instructional strategies could be efficient and lead to persistent knowledge. This is especially true when learning activities involve the use of technologies, as they are more likely to be inattentive due to exposure to other sources of distraction. This may affect the acquisition of knowledge [5].

This paper deals with the issue of attention monitoring, with the aim of proving a non-intrusive and non-invasive system, reliable and easy tool that can be used freely in schools, without changing or interfering with the established working routines.

This paper is organized as follows. In the next Section the state of art of technology in learning and ambient intelligent where scientific literature is reviewed. Section 3 contains the proposed architecture, section 4 presented the methodology applied with data acquisition and in section 5 preliminary results are presented. Finally, in Section 6 some conclusions of this work are presented and future directions are suggested.

\section{State of Art}

Learning is a skill used in all of the human's lifetime, and it can be defined as the art or process of acquiring knowledge or skill. It is very linked with attention as without attention the learning ability is severally impaired. Another theory associated is: less attention causes less information retained by the brain [6].

\subsection{Technology in Learning}

Technology is shown to help low performing students to increase their results and attitudes towards the classroom [6]. The stimulus provided by the technology is more advantageous than disadvantageous [5].

Although the difficulties of having technology in classroom are disappearing some strongest factors can proved that they can still be felt. This is especially true when we consider the lack of training and disbelief in the role of technology, the necessary time spent, and the costs of equipment. 
The classroom contains not only students but also teachers, so the use of technology in learning also changes the role of these teachers. It is required that the teacher have more training, time available to study and explain the technology that will be used in the classroom.

There are many technologies that can be used to learning like online collaboration tools, presentation software, tablet, course management tools, clickers and smartphones, lecture-capture tools, and audio tools. These technologies have many advantages to help students to learn faster and better, by allowing teacher to adapt the students learning style. However, there are many distractions that can disrupt attention present in these technologies. With the emergence of social networks and games, it can be very difficult for the teacher to know if the students are really working on the pre-defined tasks or if they are wasting their time and attention on other tasks [7].

\subsection{Ambient Intelligent}

The collection, storage, management, and anticipation of contextual information about the user to support decision-making constitute some of the key operations in most AmI systems.

An AmI system is an environment in which technology is embedded, hidden in the background, sensitive, adaptive and responsive to the presence of people and objects. This system also preserves security and privacy while using information when needed and with an appropriate context [8].

In the case of this work, adaptive systems aim at supporting and enhancing a student's learning process [9]. In their supply of adaptability, adaptive systems generally consider the student's knowledge, background, interest, goals, targets and/or choices [10].

\section{The Proposed Architecture}

In this section it will be presented the proposed architecture approach. The modules will be developed following the notions of encapsulation, inheritance, polymorphism, association, and abstract classes. The technologies applied for this system are JavaFX, SQL, and REST. The architecture is presented in Figure 1.

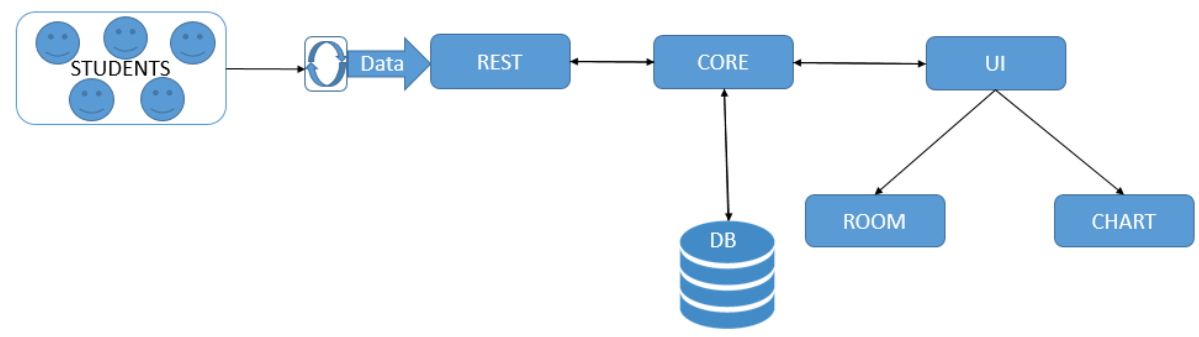

Fig. 1. The proposed architecture of the AmI system. 
The proposed architecture contains a rest service (REST) that receives data in real time from computer student's. This data contains the peripheral behaviors biometrics student's features. This rest service (REST) sends the data received to the core module (CORE) of the application. In this field, all processing will be made and compare with previous data stored in database (DB). Finally, the database (DB) will be updated.

Then there are some graphical modules that allow showing the information in an intuitive way to the user. These graphical modules stand out the user interface (UI) that allows the teacher to control the application. This graphical module user interface (UI) is composed by a module that allow the creation of charts (CHART) and the layer that allows the creation of virtual classrooms (ROOM) so that the teacher may view intuitively the students behavior.

\subsection{REST}

As previously mentioned, this module will deal with the transportation of the data of the peripheral behaviors biometrics student's features. The requests have a defined Json format that allows exchanging information with the classifier.

This application will be send information about what should be considered as a task and which students it will be applied to. Then it'll receive the attention data about the global class and each student. The technology used in the making of this layer is REST and Java.

\subsection{CORE}

This is the module where all the business logic will be located, so all the required processing will be done in this layer. This module provides powerful tools for performing analytics and statically analysis in real-time. This layer simplifies the code and limits resources requirements. It is important that in this process some values are filtered to eliminate possible negative effects on the analysis. The system calculates, at regular intervals, an estimation of the general level of performance and attention of each student.

Here it will be tested for input errors from the application users and received errors from the received data, it will be also the module responsible for managing the sync time with the REST layer. This module it will be a Java based module.

\subsection{DB}

In this module the raw data received from the data generating devices is stored in a data store engine. Here it is acknowledged the supporting database and it will store the students' attention profiles, the class attention profiles and the previous made classroom tasks, it will also store the information linking the students to the correct class and the possible classrooms plants. 
The classifier will also interact directly with the database, this is helpful to reduce the needed quantity of data to pass through rest and speed up that process. For the construction of this module it will be used MySQL for this module.

\section{$3.4 \quad$ UI}

The system will need to interact with the teacher, so this module will provide that. It will contain the window to order: introduction of the classroom tasks; ordering to starting collect data; button to authorization to create news classrooms; and button to choses students, and tasks.

This module needs to follows the known aspect of usual applications so that it would be easy for the teacher to use. For the construction of this module it will be made using JavaFX.

\subsection{CHART}

This module will allow the teacher to see the data into charts that help him to concentrate information and study the student's behavior in classroom, instead of the attention, and other reference indicator being displayed in a non-intuitive and non-invasive way.

This layer allows the teachers to make all the charts that they feel the need to, so it would have to be highly adaptive to every user and it is built with JavaFX.

\subsection{ROOM}

Here it will be made a classroom designer, to allow teacher to display the student's attention so that he can see in little time what students or sectors of the classroom are not attentive.

This module needs to allow the teacher to design where the seat students of the room where in the classroom. This system has some pre-designed room, which the teachers can use. If the design room proposed didn't exist the teacher can created or adopted a notation to persist the created rooms, and it is built with JavaFX.

\section{Methodology}

In this work we compare the class from the sciences and technology course while performing an activity based on Microsoft Excel at the High School of Caldas das Taipas, Guimarães, Portugal. We want to determine how the class reacts during the lesson and the effect on mouse and keyboard dynamics, and attention level.

For this purpose one group of 28 (19 girls and 9 boys) students were selected to participate in this experience. Their average age is seventeen years old. In the lesson, they have access to an individual computer and 100 minutes to complete a task. Students received, at the beginning of the lesson, a document with the goals of the task. 


\subsection{Data Acquisition}

The first stage of the proposed system is the collection of the relevant data. The data collected by the logger application characterizing the students' interaction patterns is aggregated in a server to which the logger application connects after the student logs in. The privacy of the students is ensured, since the necessary data that is collected to perform the login and the registration are an ID that does not personally identify of the student. They also need a password, and specify the gender.

This application runs in the background, which makes the data acquisition process, a completely transparent one from the point of view of the student. The system was developed to acquire data from normal working routines, compiling information from the students' activities through the mouse and the keyboard, which act as sensors. The Mouse and Keyboard Sensing layers are responsible for capturing information describing the behavioral patterns of the students, and receiving data from the events generated by their mouse and keyboard [11]. These data are further processed, stored and then used to calculate the values of the behavioral biometrics.

As previously mentioned [11], the early version of this system acquired data describing the interaction of the user with the computer in terms of the mouse and the keyboard.

This raw data includes all the important interaction events: when keys were pressed down or released, when the mouse moved (and where to), when clicks started or ended and when the user switched to a given application. This data is then transformed by the server as described in [11], to generate 15 features that describe the performance of the interaction with the computer.

\section{$5 \quad$ Preliminary Results}

During the lesson with the class, the monitoring system was used to assess the interaction of the students with the computer and to quantify their level of attentiveness as well. To quantify attentiveness the following methodology was followed. Asides from capturing the interaction of the students with the computer, the AmI system also registers the applications with which students are interacting.

Attention is calculated at regular intervals, as configured by the teacher. The teacher may also want to assess, in real-time or a posteriori, the evolution of attention of the whole class. If necessary, the teacher may also click on a student to analyze the temporal evolution of the attention for that specific student during the class. Figure 2 presents the evolution of attention for four students in the lesson.

Theoretically, the lesson began at 8:15 a.m. and finished at 9:55 a.m. However, the students need to set down and the teacher must explain the content of the lesson. After that the student need to turn on the computer, open the session, and run the application.

In case of Figure 2 we have four students that were monitoring the level of attention. In these cases, the background application received data after 24 minutes of the lesson had begun. This mean that students only begin to open the application task at 8:39 a.m., although there are a student that only begun after 32 minutes, at 8:47a.m.. 
After finished the task, the student turn off the computer, before the lesson had finished. Some students have finished the task in 50 minutes after the lesson begun at 9:05 a.m., and other after 66 minutes at 9:21 a.m..

In term of attentiveness, we can see that each one reacts differently and had different evolution of the level of attention during the lesson. For example the student 12421000, has a good level of attention until the minute 41, after that his level of attention in the task as decrease continuous until a lower level of attentiveness (around 30\%).
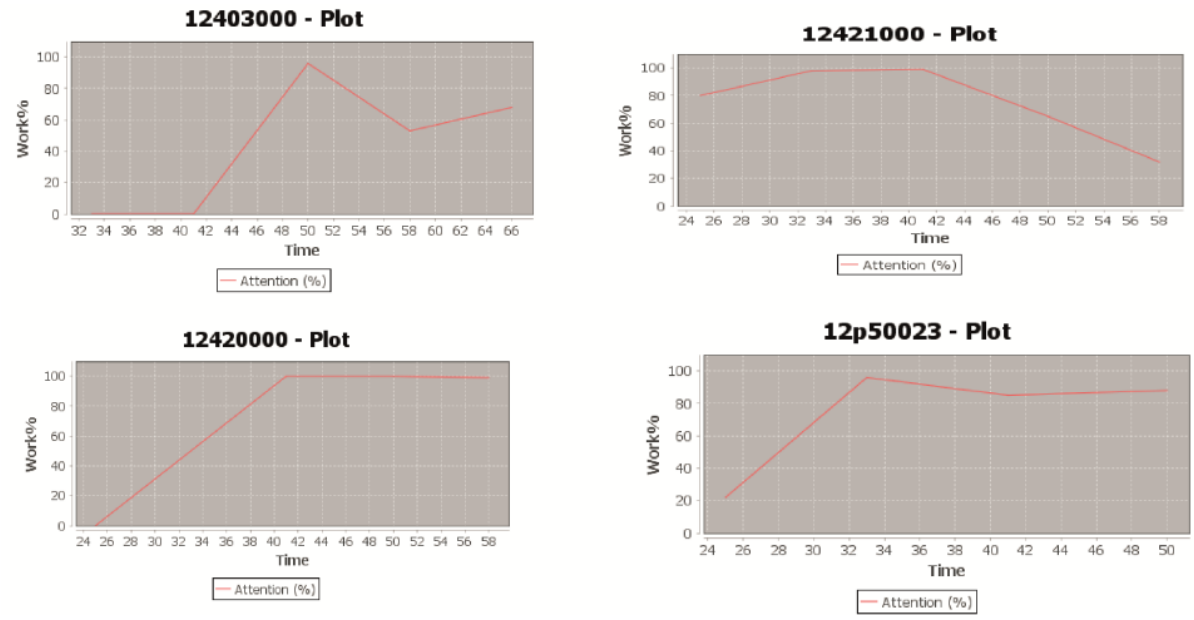

Fig. 2. Evolution of the level of attention for four students.

\section{Conclusions and Future Directions}

The work developed so far resulted in a very useful system for the teachers, which can monitor, in real-time, the level of attention of their students. However, in this moment, the architecture of the proposed AmI system can only analyze the student's level of attention from the percentage of time spent interacting with work-related applications and don't compared with the biometrics behaviors captured of the students. It also necessary having a larger amount of data in order to analyze the profile of students and predict how they react in different types of lessons.

With the proposed system it is possible to detect potentially negative factors dynamically and non-intrusively, making it possible to foresee negative situations, and to take actions to mitigate them. This may, in turn, minimize issues such as stress and anxiety, which can negatively influence the students' results and are closely related to the occurrence of conflicts.

In future work we will implement a tighter integration between the monitoring of the interaction of the users with the keyboard and the mouse (biometrics behaviors) and the performed tasks. With the data of biometrics behaviors it'll be possible to know all the actions that each user performed both with the mouse or the keyboard, 
and at what time. We will thus generate a new feature that will quantify the level of activity of each user throughout time. This new feature will allow a more contextualized analysis of attention, improving the performance of its classification and quantification.

\section{Acknowledgements}

This work has been supported by COMPETE: POCI-01-0145-FEDER-007043 and FCT - Fundação para a Ciência e Tecnologia within the Project Scope: $\mathrm{UID} / \mathrm{CEC} / 00319 / 2013$.

\section{References}

1. Carroll,J. M. (1997). Human-Computer Interaction: Psychology as a Science of Design. Annual Review of Psychology, 48, 61-83. doi:10.1146/annurev.psych.48.1.61.

2. Milrad, M., Wong, L. H., Sharples, M., Hwang, G. - J., Looi, C. - K., Ogata, H. (2013). Seamless Learning: An International Perspective on Next Generation Technology Enhanced Learning. Book chapter in Z. L. Berge \& L. Y. Muilenburg (eds.) Handbook of Mobile Learning, 95-108, New York: Routledge.

3. A. Pimenta, S. Gonçalves, D. Carneiro, F. Riverola, P. Novais, "Mental Workload Management as a Tool in e-Learning Scenarios," 2014

4. Hwang, K., Yang, C (2009). Automated Inattention and Fatigue Detection System in Distance Education for Elementary School Students. Journal of Educational Technology 12, 22-35.

5. Durães, D., Jiménez, A., Bajo, J., Novais, P. (2016). Monitoring Level Attention Approach in Learning Activities. Advances in Intelligent System and Computing, 478, 33-40. Doi: 10.1007/978-3-319-40165-2_4.

6. McNeely, B. (2005). Using Technology as a Learning Tool, Not Just the Cool New Thing. Educating the Net Generation, $4.1-4.10$.

7. Muir-Herzig, R. (2003). Technology and Its Impact in the Classroom. DOI: 10.1016/S0360-1315(03)00067-8.

8. Weber, W., Rabaey, J. M., Aarts, E. (2005). Ambient Intelligence, Springer, 1-2.

9. Neto, W. B., Gauthier, F., Nassar, S. M. (2005). An Adaptive e-Learning Model for the Semantic Web. International Workshop on Applications of Semantic Web technologies for E-Learning. Banff, Canada, 63-64.

10. Khan, F. A., Grap, S., Weippl, E. R., Tjoa, A. M. (2010). Identifying and Incorporating Affective Sate and Learning Styles in Web-Based Learning Management Systems. Interaction Design and Architecture (s) Journal - IXD\&A, N. 9-10, 85-103.

11. Durães, D., Carneiro, D., Bajo, J., Novais, P. (2016). Using Computer Peripheral Devices to measure attentiveness. Advances in Intelligent System and Computing, 147-155. 